\title{
Finite genus solutions for Geng hierarchy
}

Zhu Li

To cite this article: Zhu Li (2018) Finite genus solutions for Geng hierarchy, Journal of Nonlinear Mathematical Physics 25:1, 54-65, DOI:

https://doi.org/10.1080/14029251.2018.1440742

To link to this article: https://doi.org/10.1080/14029251.2018.1440742

Published online: 04 January 2021 


\title{
Finite genus solutions for Geng hierarchy
}

\author{
Zhu Li \\ School of Mathematics and Statistics, Xinyang Normal University \\ 237 Nanhu Road, Xinyang, Henan 464000, China \\ lizhu2020@126.com
}

Received 25 October 2016

Accepted 18 August 2017

\begin{abstract}
The Geng hierarchy is derived with the aid of Lenard recursion sequences. Based on the Lax matrix, a hyperelliptic curve $\mathscr{K}_{n+1}$ of arithmetic genus $n+1$ is introduced, from which meromorphic function $\phi$ is defined. The finite genus solutions for Geng hierarchy are achieved according to asymptotic properties of $\phi$ and the algebro-geometric characters of $\mathscr{K}_{n+1}$.
\end{abstract}

Keywords: Hyperelliptic curve; meromorphic function; finite genus solutions.

2000 Mathematics Subject Classification: 35Q51, 35C08, 14H55

\section{Introduction}

The soliton equations describe various nonlinear phenomena in natural and applied sciences such as fluid dynamics, plasma physics, optical fibers and other sciences. It is of great importance to solve nonlinear soliton equations from both theoretical and practical points of view. Due to the nonlinearity of soliton equations, it is a difficult job for us to determine whatever exact solutions to soliton equations, but with the development of soliton theory several systematic methods has been developed to obtain explicit solutions of soliton equations, such as the inverse scattering transformation [1], the Hirota bilinear transformation [2], the Bäcklund and the Darboux transformation [3,4], the algebro-geometric method [5], the nonlinearization approach of Lax pairs [6], the homogeneous balance method [7], etc [8-12].

The nonlinear diffusion equation [13-15]

$$
u_{t}=\left(\frac{u_{x}}{u^{2}}\right)_{x}
$$

which has a lot of applications in plasma physics, laser physics, semiconductor physics, astrophysics, population dynamics in biology and helium combustion process in chemistry, can be obtained in the following equations in case of $v=1$ [16]

$$
\left\{\begin{array}{l}
u_{t}=\left(\frac{1}{u^{2}}\right)_{x}-\left(\frac{v^{2}}{u^{2}}\right)_{x}-\left(\frac{v}{u}\right)_{x x}, \\
v_{t}=\frac{1}{2}\left(\frac{1}{u^{2}}\right)_{x x}-\frac{1}{2}\left(\frac{v^{2}}{u^{2}}\right)_{x x} .
\end{array}\right.
$$

In this paper we will concentrate primarily on constructing the finite genus solutions of the entire Geng hierarchy related to (1.2) based on the approaches in Refs. [17-32]. The finite genus solutions 
are associated to nonłlinear flows in the Jacobian of a hyperelliptic curve. This phenomenon is connected to the existence of integrable hierarchies with nonłlinear dependence on the spectral parameter. Such problem was first considered in Refs. [24] and [25]. The algebraic geometric approach was proposed in [26]. Key examples are the Camassa-Holm [27] and Harry dym equations [28] whose algebraic geometric solutions produce nonłlinear flows in the generalized Jacobian of hyperelliptic curves in Refs. [29] and [30]. After separation of variables, the appearance of nonflinear flows in the (generalized) Jacobians of algebraic curves, also appears in ODEs and was first considered in Refs [31] and [32].

The outline of this paper is as follows. In Section 2, we obtain the coupled diffusion equation hierarchy based on Lenard recursion sequences. In Section 3, with the aid of Lax matrix we shall introduce hyperelliptic curve $\mathscr{K}_{n+1}$ of arithmetic genus $n+1$. In Section 4, we define the meromorphic function $\phi$ and investigate the asymptotic properties of $\phi$. Moreover, we construct the finite genus solutions of the whole hierarchy by use of the Riemann theta functions according to the asymptotic properties of $\phi$ and the algebro-geometric characters of $\mathscr{K}_{n+1}$.

\section{Hierarchy of nonlinear evolution equations}

In this section, we shall derive the Geng hierarchy associated with the $2 \times 2$ spectral problem [16]

$$
\varphi_{x}=U \varphi, \quad \varphi=\left(\begin{array}{l}
\varphi_{1} \\
\varphi_{2}
\end{array}\right), \quad U=\left(\begin{array}{cc}
\lambda u & v-1 \\
\lambda(v+1)-\lambda u
\end{array}\right)
$$

where $u$ and $v$ are two potentials, and $\lambda$ is a constant spectral parameter. To this end, we first introduce the Lenard recursion sequences

$$
K L_{j}=J L_{j+1}, \quad L_{j}=\left(a_{j}, b_{j}\right)^{T}, \quad j \geq 0
$$

with three starting points

$$
L_{0}=\left(\frac{1-v^{2}}{2 u^{2}}, \frac{v}{u}\right)^{T}, \quad \bar{L}_{0}=(1,0)^{T}, \quad \tilde{L}_{0}=(0,1)^{T}
$$

where $K$ and $J$ are two operators defined by

$$
K=\left(\begin{array}{cc}
2 \partial & -\partial^{2} \\
\partial^{2} & 0
\end{array}\right), \quad J=2\left(\begin{array}{cc}
\partial u \partial^{-1} u \partial & \partial u \partial^{-1} v \partial \\
\partial v \partial^{-1} u \partial \partial \nu \partial^{-1} v \partial-\partial
\end{array}\right), \quad \partial \partial^{-1}=\partial^{-1} \partial=1 .
$$

It is easy to see that $\operatorname{Ker} J=\left\{\alpha_{0} L_{0}+\bar{\alpha}_{0} \bar{L}_{0}+\tilde{\alpha}_{0} \tilde{L}_{0} \mid \forall \alpha_{0}, \bar{\alpha}_{0}, \tilde{\alpha}_{0} \in \mathbb{R}\right\}$. Hence $L_{j}$ are uniquely determined by the recursion relation (2.2) up to a term const. $L_{0}+$ const. $\bar{L}_{0}+$ const. $\tilde{L}_{0}$, which is always assumed to be zero. Assume that the eigenfunction $\varphi$ satisfies an auxiliary problem

$$
\boldsymbol{\varphi}_{t_{m}}=V^{(m)} \boldsymbol{\varphi}, \quad V^{(m)}=\left(\begin{array}{cc}
V_{11}^{(m)} & V_{12}^{(m)} \\
V_{21}^{(m)} & -V_{11}^{(m)}
\end{array}\right)
$$


where $V_{11}^{(m)}, V_{12}^{(m)}, V_{21}^{(m)}$ are defined as follows;

$$
\begin{aligned}
& V_{11}^{(m)}=\sum_{j=0}^{m}\left(-\partial b_{j}-2 u \partial^{-1} u \partial a_{j} \lambda-2 u \partial^{-1} v \partial b_{j} \lambda\right) \lambda^{m+1-j}, \\
& V_{12}^{(m)}=\sum_{j=0}^{m}\left(\partial a_{j}+2 \partial^{-1} u \partial a_{j} \lambda-2 v \partial^{-1} u \partial a_{j} \lambda+2 \partial^{-1} v \partial b_{j} \lambda-2 v \partial^{-1} v \partial b_{j} \lambda\right) \lambda^{m-j}, \\
& V_{21}^{(m)}=\sum_{j=0}^{m}\left(\partial a_{j}-2 \partial^{-1} u \partial a_{j} \lambda-2 v \partial^{-1} u \partial a_{j} \lambda-2 \partial^{-1} v \partial b_{j} \lambda-2 v \partial^{-1} v \partial b_{j} \lambda\right) \lambda^{m+1-j} .
\end{aligned}
$$

Then the compatibility condition of (2.1) and (2.5) yields the zero curvature equation, $U_{t_{m}}-V_{x}^{(m)}+$ $\left[U, V^{(m)}\right]=0$, which is equivalent to a class of nonlinear evolution equations

$$
u_{t_{m}}=2 a_{m, x}-b_{m, x x}, \quad v_{t_{m}}=a_{m, x x} .
$$

The first two nontrivial flows in (2.7) are (1.2) and

$$
\begin{aligned}
u_{t}= & \frac{1}{u}\left(\frac{1-v^{2}+v u_{x}-u v_{x}}{u^{3}}\right)_{x}-\frac{v}{u}\left(\frac{2 u_{x}-v^{2} u_{x}+u v v_{x}+v-v^{3}}{u^{3}}\right)_{x} \\
& -\frac{1}{2}\left(\frac{2 u_{x}-v^{2} u_{x}+u v v_{x}+v-v^{3}}{u^{3}}\right)_{x x}, \\
v_{t}= & \frac{1}{2}\left(\frac{1}{u}\left(\frac{1-v^{2}+v u_{x}-u v_{x}}{u^{3}}\right)_{x}-\frac{v}{u}\left(\frac{2 u_{x}-v^{2} u_{x}+u v v_{x}+v-v^{3}}{u^{3}}\right)_{x}\right)_{x} .
\end{aligned}
$$

\section{Hyperelliptic curve}

Let $\chi=\left(\chi_{1}, \chi_{2}\right)^{T}$ and $\psi=\left(\psi_{1}, \psi_{2}\right)^{T}$ be two basic solutions of (2.1) and (2.5). We introduce a Lax matrix

$$
W=\frac{1}{2}\left(\chi \psi^{T}+\psi \chi^{T}\right)\left(\begin{array}{cc}
0 & -1 \\
1 & 0
\end{array}\right)=\left(\begin{array}{cc}
G & F \\
H & -G
\end{array}\right)
$$

which satisfies the Lax equations

$$
W_{x}=[U, W], \quad W_{t_{m}}=\left[V^{(m)}, W\right] .
$$

Therefore, $\operatorname{det} W$ is a constant independent of $x$ and $t_{m}$. Equation (3.2) can be written as

$$
\begin{aligned}
G_{x} & =(v-1) H-\lambda(v+1) F, \\
F_{x} & =2 \lambda u F-2(v-1) G, \\
H_{x} & =2 \lambda(v+1) G-2 \lambda u H,
\end{aligned}
$$

and

$$
\begin{aligned}
G_{t_{m}} & =V_{12}^{(m)} H-V_{21}^{(m)} F, \\
F_{t_{m}} & =2\left(V_{11}^{(m)} F-V_{12}^{(m)} G\right), \\
H_{t_{m}} & =2\left(V_{21}^{(m)} G-V_{11}^{(m)} H\right) .
\end{aligned}
$$


Suppose functions $F, G$ and $H$ are finite-order polynomials in $\lambda$

$$
G=\sum_{j=0}^{n+1} G_{j} \lambda^{n+2-j}, \quad F=\sum_{j=0}^{n+1} F_{j} \lambda^{n+1-j}, \quad H=\sum_{j=0}^{n+1} H_{j} \lambda^{n+2-j},
$$

where

$$
\begin{aligned}
& G_{0}=-2 u \partial^{-1} u \partial c_{0}-2 u \partial^{-1} v \partial d_{0}, \quad F_{0}=2(1-v) \partial^{-1} u \partial c_{0}+2(1-v) \partial^{-1} v \partial d_{0}, \\
& H_{0}=-2(1+v) \partial^{-1} u \partial c_{0}-2(1+v) \partial^{-1} v \partial d_{0}, \quad F_{n+1}=H_{n+1}=c_{n, x}, \\
& G_{j}=-\partial d_{j-1}-2 u \partial^{-1} u \partial c_{j}-2 u \partial^{-1} v \partial d_{j}, \quad 1 \leq j \leq n, \quad G_{n+1}=-d_{n, x}, \\
& F_{j}=\partial c_{j-1}+2(1-v) \partial^{-1} u \partial c_{j}+2(1-v) \partial^{-1} v \partial d_{j}, \quad 1 \leq j \leq n, \\
& H_{j}=\partial c_{j-1}-2(1+v) \partial^{-1} u \partial c_{j}-2(1+v) \partial^{-1} v \partial d_{j}, \quad 1 \leq j \leq n .
\end{aligned}
$$

Substituting (3.5) and (3.6) into (3.3) yields

$$
\begin{aligned}
& K E_{j}=J E_{j+1}, \quad J E_{0}=0, \\
& K E_{n}=0,
\end{aligned}
$$

where $E_{j}=\left(c_{j}, d_{j}\right)^{T}, 0 \leq j \leq n-1$. It is easy to see that the equation $J E_{0}=0$ has a special general solution

$$
E_{0}=L_{0}
$$

By induction, we obtain from recursive relations (3.7) and (2.2) that

$$
E_{k}=\sum_{j=0}^{k} \alpha_{j} L_{k-j}, \quad 0 \leq k \leq n,
$$

which are special solutions of (3.7), where $\alpha_{1}, \alpha_{2}, \ldots, \alpha_{k}$ are constants of integration and $\alpha_{0}=1$. Moreover, from (3.8) we can get

$$
c_{n}=\beta_{0} x+\beta_{1}, \quad d_{n}=\beta_{0} x^{2}+\beta_{2} x+\beta_{3},
$$

where $\beta_{0}, \beta_{1}, \beta_{2}, \beta_{3}$ are constants of integration.

Since $\operatorname{det} W$ is a $(2 n+4)$ th-order polynomial in $\lambda$, whose coefficients are constants independent of $x$ and $t_{m}$, we have

$$
-\operatorname{det} W=G^{2}+F H=4 \lambda \prod_{j=1}^{2 n+3}\left(\lambda-\lambda_{j}\right)=4 R(\lambda),
$$

one is naturally led to introduce the hyperelliptic curve $\mathscr{K}_{n+1}$ of arithmetic genus $n+1$ defined by

$$
\mathscr{K}_{n+1}: y^{2}-R(\lambda)=0 .
$$

The curve $\mathscr{K}_{n+1}$ can be compactified by joining two points at infinity, $P_{\infty \pm}$, where $P_{\infty+} \neq P_{\infty-}$. For notational simplicity the compactification of the curve $\mathscr{K}_{n+1}$ is also denoted by $\mathscr{K}_{n+1}$. Here we assume that the zeros $\lambda_{j}$ of $R(\lambda)$ in (3.12) are mutually distinct. Then the hyperelliptic curve $\mathscr{K}_{n+1}$ becomes nonsingular and irreducible. 
We write $F$ and $H$ as finite products which take the form

$$
F=\frac{2(1-v)}{u} \prod_{j=1}^{n+1}\left(\lambda-\mu_{j}\right), \quad H=-\frac{2(1+v)}{u} \lambda \prod_{j=1}^{n+1}\left(\lambda-v_{j}\right)
$$

where $\left\{\mu_{j}\right\}_{j=1}^{n+1}$ and $\left\{v_{j}\right\}_{j=1}^{n+1}$ are called elliptic variables. According to the definition of $\mathscr{K}_{n+1}$, we can lift the roots $\mu_{j}$ and $v_{j}$ to $\mathscr{K}_{n+1}$ by introducing

$$
\begin{aligned}
& \hat{\mu}_{j}\left(x, t_{m}\right)=\left(\mu_{j}\left(x, t_{m}\right),-\frac{1}{2} G\left(\mu_{j}\left(x, t_{m}\right), x, t_{m}\right)\right), \quad j=1, \ldots, n+1, \\
& \hat{v}_{j}\left(x, t_{m}\right)=\left(v_{j}\left(x, t_{m}\right), \frac{1}{2} G\left(v_{j}\left(x, t_{m}\right), x, t_{m}\right)\right), \quad j=1, \ldots, n+1,
\end{aligned}
$$

where $\left(x, t_{m}\right) \in \mathbb{R}^{2}$.

From the following lemma, we can explicitly represent $\alpha_{l}(0 \leq l \leq n)$ by the constants $\lambda_{1}, \ldots, \lambda_{2 n+3}$.

\section{Lemma 3.1.}

$$
\alpha_{l}=c_{l}(\underline{\Lambda}), \quad l=0, \ldots, n,
$$

where

$$
\begin{gathered}
\underline{\Lambda}=\left(\lambda_{1}, \ldots, \lambda_{2 n+3}\right), \quad c_{0}(\underline{\Lambda})=1, \quad c_{1}(\underline{\Lambda})=-\frac{1}{2} \sum_{j=1}^{2 n+3} \lambda_{j}, \ldots, \\
c_{l}(\underline{\Lambda})=-\sum_{\substack{j_{1}, \ldots, j_{2 n+3}=0 \\
j_{1}+\ldots+j_{2 n+3}=l}}^{l} \frac{\left(2 j_{1}\right) ! \ldots\left(2 j_{2 n+3}\right) ! \lambda_{1}^{j_{1}} \ldots \lambda_{2 n+3}^{j_{2 n+3}}}{2^{2 l}\left(j_{1} !\right)^{2} \ldots\left(j_{2 n+3} !\right)^{2}\left(2 j_{1}-1\right) \ldots\left(2 j_{2 n+3}-1\right)} .
\end{gathered}
$$

Proof. Assume that

$$
\hat{F}_{j}=\left.F_{j}\right|_{\alpha_{1}=\ldots=\alpha_{j}=0}, \quad \hat{H}_{j}=\left.H_{j}\right|_{\alpha_{1}=\ldots=\alpha_{j}=0}, \quad \hat{G}_{j}=\left.G_{j}\right|_{\alpha_{1}=\ldots=\alpha_{j}=0} .
$$

It will be convenient to introduce the notion of a degree, $\operatorname{deg}($.$) , to effectively distinguish between$ homogeneous and nonhomogeneous quantities. Define

$$
\operatorname{deg}(u)=-1, \quad \operatorname{deg}(v)=0, \quad \operatorname{deg}\left(\partial_{x}\right)=1,
$$

thus from (3.7) it can be implied that

$$
\operatorname{deg}\left(\hat{F}_{k}\right)=\operatorname{deg}\left(\hat{H}_{k}\right)=2 k+1, \quad \operatorname{deg}\left(\hat{G}_{k}\right)=2 k, \quad k \in \mathbb{N}_{0} .
$$

Temporarily fixed the branch of $R(\lambda)^{1 / 2}$ as $\lambda^{n+2}$ near infinity, $R(\lambda)^{-1 / 2}$ has the following expansion

$$
R(\lambda)^{-1 / 2} \underset{\lambda \rightarrow \infty}{=} \sum_{l=0}^{\infty} \hat{c}_{l}(\underline{\Lambda}) \lambda^{-n-2-l}
$$

where

$$
\begin{gathered}
\underline{\Lambda}=\left(\lambda_{1}, \ldots, \lambda_{2 n+3}\right), \quad \hat{c}_{0}(\underline{\Lambda})=1, \quad \hat{c}_{1}(\underline{\Lambda})=\frac{1}{2} \sum_{j=1}^{2 n+3} \lambda_{j}, \ldots, \\
\hat{c}_{l}(\underline{\Lambda})=\sum_{\substack{j_{1}, \ldots, j_{2 n+3}=0 \\
j_{1}+\ldots+j_{2 n+3}=l}}^{l} \frac{\left(2 j_{1}\right) ! \ldots\left(2 j_{2 n+3}\right) ! \lambda_{1}^{j_{1} \ldots} \ldots \lambda_{2 n+3}^{j_{2 n+3}}}{2^{2 l}\left(j_{1} !\right)^{2} \ldots\left(j_{2 n+3} !\right)^{2}}
\end{gathered}
$$


Dividing $F(\lambda), H(\lambda), G(\lambda)$ by $R(\lambda)^{1 / 2}$ near infinity respectively, we obtain

$$
\begin{aligned}
& \frac{F(\lambda)}{R(\lambda)^{1 / 2}} \underset{\lambda \rightarrow \infty}{=} \sum_{l=0}^{\infty} \hat{c}_{l}(\underline{\Lambda}) \lambda^{-n-2-l} \sum_{l=0}^{n+1} F_{l} \lambda^{n+1-l}=\sum_{l=0}^{\infty} \check{F}_{l} \lambda^{-l-1}, \\
& \frac{H(\lambda)}{R(\lambda)^{1 / 2}} \underset{\lambda \rightarrow \infty}{=} \sum_{l=0}^{\infty} \hat{c}_{l}(\underline{\Lambda}) \lambda^{-n-2-l} \sum_{l=0}^{n+1} H_{l} \lambda^{n+2-l}=\sum_{l=0}^{\infty} \check{H}_{l} \lambda^{-l}, \\
& \frac{G(\lambda)}{R(\lambda)^{1 / 2}} \underset{\lambda \rightarrow \infty}{=} \sum_{l=0}^{\infty} \hat{c}_{l}(\underline{\Lambda}) \lambda^{-n-2-l} \sum_{l=0}^{n+1} G_{l} \lambda^{n+2-l}=\sum_{l=0}^{\infty} \check{G}_{l} \lambda^{-l},
\end{aligned}
$$

for some coefficients $\breve{F}_{l}, \breve{F}_{l}, \breve{G}_{l}$ to be determined next. From (3.3) and (3.24), we have

$$
\begin{aligned}
& \check{G}_{k, x}=(v-1) \check{H}_{k}-(v+1) \check{F}_{k}, \\
& \check{F}_{k, x}=2 u \breve{F}_{k+1}-2(v-1) \check{G}_{k+1}, \\
& \check{H}_{k, x}=2(v+1) \check{G}_{k+1}-2 u \breve{H}_{k+1},
\end{aligned}
$$

for $k \in \mathbb{N}_{0}$. The initial values of $\check{F}_{0}, \check{H}_{0}$ and $\check{G}_{0}$ have been chosen as $\check{F}_{0}=\frac{2(1-v)}{u}, \check{H}_{0}=-\frac{2(1+v)}{u}, \check{G}_{0}=$ -2 such that $\breve{F}_{0}=\hat{F}_{0}, \breve{H}_{0}=\hat{H}_{0}$ and $\breve{G}_{0}=\hat{G}_{0}$. Moreover, we can prove inductively that

$$
\operatorname{deg}\left(\check{F}_{k}\right)=\operatorname{deg}\left(\check{H}_{k}\right)=2 k+1, \quad \operatorname{deg}\left(\check{G}_{k}\right)=2 k, \quad k \in \mathbb{N}_{0} .
$$

Hence, $\breve{F}_{l}, \breve{H}_{l}$ and $\breve{G}_{l}$ are equal to $\hat{F}_{l}, \hat{H}_{l}$ and $\hat{G}_{l}$ respectively for all $l \in \mathbb{N}_{0}$. Thus we proved

$$
\frac{F(\lambda)}{R(\lambda)^{1 / 2}}=\sum_{l=0}^{\infty} \hat{F}_{l} \lambda^{-l-1}, \quad \frac{H(\lambda)}{R(\lambda)^{1 / 2}}=\sum_{l=0}^{\infty} \hat{H}_{l} \lambda^{-l}, \quad \frac{G(\lambda)}{R(\lambda)^{1 / 2}}=\sum_{l=0}^{\infty} \hat{G}_{l} \lambda^{-l} .
$$

Considering

$$
R(\lambda)^{1 / 2} \underset{\lambda \rightarrow \infty}{=} \sum_{l=0}^{\infty} c_{l}(\underline{\Lambda}) \lambda^{n+2-l}
$$

a comparison of the coefficients of $\lambda^{-k}$ in the following equation

$$
1=R(\lambda)^{1 / 2} \times R(\lambda)^{-1 / 2}=\left(\sum_{l=0}^{\infty} c_{l}(\underline{\Lambda}) \lambda^{n+2-l}\right)\left(\sum_{l=0}^{\infty} \hat{c}_{l}(\underline{\Lambda}) \lambda^{-n-2-l}\right)
$$

yields

$$
\sum_{l=0}^{k} c_{k-l}(\underline{\Lambda}) \hat{c}_{l}(\underline{\Lambda})=\delta_{k, 0}, \quad k \in \mathbb{N}_{0}
$$

Therefore, we compute that

$$
\sum_{m=0}^{k} c_{k-m}(\underline{\Lambda}) \hat{F}_{m}=\sum_{m=0}^{k} c_{k-m}(\underline{\Lambda}) \sum_{l=0}^{m} F_{l} \hat{c}_{m-l}(\underline{\Lambda})=\sum_{l=0}^{k} F_{l} \sum_{p=0}^{k-l} c_{k-l-p}(\underline{\Lambda}) \hat{c}_{p}(\underline{\Lambda})=F_{k},
$$

where $k=0, \ldots, n$. 


\section{Finite genus solutions}

Equip the $\mathscr{K}_{n+1}$ with canonical basis cycles: $\tilde{a}_{1}, \ldots, \tilde{a}_{n+1} ; \tilde{b}_{1}, \ldots, \tilde{b}_{n+1}$, which are independent and have intersection numbers as follows

$$
\tilde{a}_{j} \circ \tilde{a}_{k}=0, \quad \tilde{b}_{j} \circ \tilde{b}_{k}=0, \quad \tilde{a}_{j} \circ \tilde{b}_{k}=\delta_{j k} .
$$

For the present, we will choose our basis as the following set [17]

$$
\tilde{\omega}_{l}=\frac{\lambda^{l-1} d \lambda}{y(P)}, \quad 1 \leq l \leq n+1,
$$

which are $n+1$ linearly independent homomorphic differentials on $\mathscr{K}_{n+1}$. Then the period matrices $A$ and $B$ can be constructed from

$$
A_{k j}=\int_{\tilde{a}_{j}} \tilde{\omega}_{k}, \quad B_{k j}=\int_{\tilde{b}_{j}} \tilde{\omega}_{k} .
$$

It is possible to show that matrices $A$ and $B$ are invertible [33,34]. Now we define the matrices $C$ and $\tau$ by $C=A^{-1}, \tau=A^{-1} B$. The matrix $\tau$ can be shown to be symmetric $\left(\tau_{k j}=\tau_{j k}\right)$, and it has positive definite imaginary part $(\operatorname{Im} \tau>0)$. If we normalize $\tilde{\omega}_{l}$ into the new basis $\omega_{j}$,

$$
\omega_{j}=\sum_{l=1}^{n+1} C_{j l} \tilde{\omega}_{l}, \quad 1 \leq j \leq n+1,
$$

then we obtain

$$
\int_{\tilde{a}_{k}} \omega_{j}=\sum_{l=1}^{n+1} C_{j l} \int_{\tilde{a}_{k}} \tilde{\omega}_{l}=\delta_{j k}, \int_{\tilde{b}_{k}} \omega_{j}=\tau_{j k}
$$

Let $\mathscr{T}_{n+1}$ be the period lattice $\mathscr{T}_{n+1}=\left\{\underline{z} \in \mathbb{C}^{n+1} \mid \underline{n}+\underline{m} \tau ; \underline{m}, \underline{n} \in \mathbb{Z}^{n+1}\right\}$. The complex torus $\mathscr{T}=\mathbb{C}^{n+1} / \mathscr{T}_{n+1}$ is called the Jacobian variety of $\mathscr{K}_{n+1}$. Now we introduce the Abel map $\underline{\mathscr{A}}(P)$ : $\operatorname{Div}\left(\mathscr{K}_{n+1}\right) \rightarrow \mathscr{T}$

$$
\underline{\mathscr{A}}(P)=\left(\int_{Q_{0}}^{P} \underline{\omega}\right)\left(\bmod \mathscr{T}_{n+1}\right), \quad \underline{\mathscr{A}}\left(\sum n_{k} P_{k}\right)=\sum n_{k} \underline{\mathscr{A}}\left(P_{k}\right),
$$

where $P, P_{k} \in \mathscr{K}_{n+1}, \underline{\omega}=\left(\omega_{1}, \ldots, \omega_{n+1}\right)$.

Let $\theta(z)$ denote the Riemann theta function associated with $\mathscr{K}_{n+1}$ [33-35]:

$$
\theta(\underline{z})=\sum_{\underline{N} \in \mathbb{Z}^{n+1}} \exp \{2 \pi i<\underline{N}, \underline{z}>+\pi i<\underline{N} \tau, \underline{N}>\},
$$

where $\underline{z}=\left(z_{1}, \cdots, z_{n+1}\right) \in \mathbb{C}^{n+1},\left\langle\underline{N}, \underline{z}>=\sum_{k=1}^{n+1} N_{k} z_{k},\left\langle\underline{N} \tau, \underline{N}>=\sum_{k, j=1}^{n+1} \tau_{k j} N_{k} N_{j}\right.\right.$. For brevity, define the function $\underline{z}: \mathscr{K}_{n+1} \times \sigma^{n+1} \mathscr{K}_{n+1} \rightarrow \mathbb{C}^{n+1}$ by

$$
\underline{z}(P, \underline{Q})=\underline{\Lambda}-\underline{\mathscr{A}}(P)+\sum_{Q^{\prime} \in \underline{Q}} \mathscr{D}\left(Q^{\prime}\right) \underline{\mathscr{A}}\left(Q^{\prime}\right),
$$


where $P \in \mathscr{K}_{n+1}, Q=\left\{Q_{1}, \cdots, Q_{n+1}\right\} \in \sigma^{n+1} \mathscr{K}_{n+1}, \sigma^{n+1} \mathscr{K}_{n+1}$ denotes the $(n+1)$ th symmetric power of $\mathscr{K}_{n+1}$, and $\underline{\Lambda}=\left(\Lambda_{1}, \ldots, \Lambda_{n+1}\right)$ is the vector of Riemann constant defined by

$$
\Lambda_{j}=\frac{1}{2}\left(1+\tau_{j j}\right)-\sum_{\substack{k=1 \\ k \neq j}}^{n+1} \int_{\widetilde{a}_{k}} \omega_{k} \int_{Q_{0}}^{P} \omega_{j}, \quad j=1, \ldots, n+1 .
$$

Without loss of generality, we choose the branch point $Q_{0}=\left(\lambda_{j_{0}}, 0\right), j_{0} \in\{1, \ldots, 2 n+3\}$ as a convenient base point, and $\lambda\left(Q_{0}\right)$ is its local coordinate.

By virtue of (3.12) and (3.13) we can define the meromorphic function $\phi\left(P, x, t_{m}\right)$ on $\mathscr{K}_{n+1}$ :

$$
\phi\left(P, x, t_{m}\right)=\frac{2 y-G}{F}=\frac{H}{2 y+G},
$$

where $P=(\lambda, y) \in \mathscr{K}_{n+1} \backslash\left\{P_{\infty \pm}\right\}$.

Lemma 4.1. Suppose that $u\left(x, t_{m}\right), v\left(x, t_{m}\right) \in C^{\infty}\left(\mathbb{R}^{2}\right)$ satisfy the hierarchy (2.7). Let $\lambda_{j} \in \mathbb{C} \backslash\{0\}$, $1 \leq j \leq 2 n+3$, and $P=(\lambda, y) \in \mathscr{K}_{n+1} \backslash\left\{P_{\infty+}, P_{\infty+}, P_{0}\right\}$, where, $P_{0}=(0,0)$. Then

$$
\phi\left(P, x, t_{m}\right) \underset{\zeta \rightarrow 0}{=}\left\{\begin{array}{l}
\frac{1+v}{2 u}+O(\zeta), \text { as } P \rightarrow P_{\infty+}, \quad \zeta=\lambda^{-1}, \\
\frac{2 u}{1-v} \zeta^{-1}+O(1), \text { as } P \rightarrow P_{\infty-}, \quad \zeta=\lambda^{-1},
\end{array}\right.
$$

and

$$
\phi\left(P, x, t_{m}\right) \underset{\zeta \rightarrow 0}{=} \zeta+O\left(\zeta^{2}\right), \text { as } P \rightarrow P_{0}, \zeta=\sigma \lambda^{\frac{1}{2}}, \sigma= \pm 1
$$

Proof. From (3.12) and (3.13), we have

$$
y \underset{\zeta \rightarrow 0}{=} \mp \zeta^{-n-2}\left(1+\frac{2 G_{0} G_{1}+F_{0} H_{0}}{8} \zeta+O\left(\zeta^{2}\right)\right) \text {, as } P \rightarrow P_{\infty \pm} \text {. }
$$

From (3.5), we obtain

$$
\begin{gathered}
G \underset{\zeta \rightarrow 0}{=} \zeta^{-n-2}\left(G_{0}+G_{1} \zeta+O\left(\zeta^{2}\right)\right), \text { as } P \rightarrow P_{\infty \pm}, \\
F \underset{\substack{\zeta \rightarrow 0 \\
=}}{ } \zeta^{-n-1}\left(F_{0}+F_{1} \zeta+O\left(\zeta^{2}\right)\right) \text {, as } P \rightarrow P_{\infty \pm} .
\end{gathered}
$$

Then according to the definition of $\phi\left(P, x, t_{m}\right)$ in (4.10), we have

$$
\begin{aligned}
\phi\left(P, x, t_{m}\right) & =\frac{2 y-G}{F} \\
& =\frac{\mp 2-G_{0}+\left(\mp \frac{2 G_{0} G_{1}+F_{0} H_{0}}{4}-G_{1}\right) \zeta+O\left(\zeta^{2}\right)}{\zeta\left(F_{0}+F_{1} \zeta+O\left(\zeta^{2}\right)\right)} \\
& =\left\{\begin{array}{l}
\frac{1+v}{2 u}+O(\zeta), \text { as } P \rightarrow P_{\infty+}, \\
\frac{2 u}{1-v} \zeta^{-1}+O(1), \text { as } P \rightarrow P_{\infty-} .
\end{array}\right.
\end{aligned}
$$


To prove (4.12), we introduce the local coordinate $\zeta=\sigma \lambda^{\frac{1}{2}}$ near $P_{0}$. Similarly we have

$$
\begin{aligned}
& y_{\zeta \rightarrow 0}=\frac{1}{2} F_{n+1} \zeta+O\left(\zeta^{3}\right) \text {, as } P \rightarrow P_{0}, \\
& G \underset{\zeta \rightarrow 0}{=} G_{n+1} \zeta^{2}+O\left(\zeta^{4}\right), \text { as } P \rightarrow P_{0} \\
& F \underset{\zeta \rightarrow 0}{=} F_{n+1}+O\left(\zeta^{2}\right) \text {, as } P \rightarrow P_{0},
\end{aligned}
$$

then (4.12) is given by virtue of (4.10) and (4.17)-(4.19).

The divisor of $\phi\left(P, x, t_{m}\right)$ is given by

$$
\left(\phi\left(P, x, t_{m}\right)\right)=\mathscr{D}_{P_{0}, \hat{v}_{1}\left(x, t_{m}\right), \ldots, \hat{v}_{n+1}\left(x, t_{m}\right)}-\mathscr{D}_{P_{\infty},}, \hat{\mu}_{1}\left(x, t_{m}\right), \ldots, \hat{\mu}_{n+1}\left(x, t_{m}\right)
$$

from the Lemma 4.1 and the definition of $\phi\left(P, x, t_{m}\right)$ in (4.10).

Let $\omega_{P_{0}, P_{\infty}}^{(3)}(P)$ denote the normalized Abelian differentials of the third kind holomorphic on $\mathscr{K}_{n+1} \backslash\left\{P_{0}, P_{\infty-}\right\}$ with simple poles at $P_{0}$ and $P_{\infty-}$ with residues \pm 1 , respectively, which can be expressed as

$$
\omega_{P_{0}, P_{\infty-}}^{(3)}(P)=\frac{1}{2 \lambda} d \lambda+\frac{1}{2 y} \prod_{j=1}^{n+1}\left(\lambda-\delta_{j}\right) d \lambda
$$

where $\gamma_{j} \in \mathbb{C}, j=1, \ldots, n+1$, are constants that are determined by

$$
\int_{\tilde{a}_{j}} \omega_{P_{0}, P_{\infty-}}^{(3)}(P)=0, \quad j=1, \ldots, n+1 .
$$

The explicit formula (4.21) then implies

$$
\omega_{P_{0}, P_{\infty}}^{(3)}(P) \underset{\zeta \rightarrow 0}{=}\left\{\begin{array}{l}
\left(\zeta^{-1}+O(1)\right) d \zeta, \text { as } P \rightarrow P_{0}, \zeta=\sigma \lambda^{\frac{1}{2}} \\
O(1) d \zeta, \text { as } P \rightarrow P_{\infty+}, \zeta=\lambda^{-1}, \\
\left(-\zeta^{-1}+O(1)\right) d \zeta, \text { as } P \rightarrow P_{\infty-}, \zeta=\lambda^{-1}
\end{array}\right.
$$

Therefore,

$$
\int_{Q_{0}}^{P} \omega_{P_{0}, P_{\infty}-}^{(3)}(P) \underset{\zeta \rightarrow 0}{=}\left\{\begin{array}{l}
\ln \zeta+\ln \left(\omega^{0}\right)+O(\zeta), \text { as } P \rightarrow P_{0} \\
\ln \left(\omega^{\infty+}\right)+O(\zeta), \text { as } P \rightarrow P_{\infty+}, \\
-\ln \zeta+\ln \left(\omega^{\infty-}\right)+O(\zeta), \text { as } P \rightarrow P_{\infty-}
\end{array}\right.
$$

for some constants $\omega^{\infty+}, \omega^{\infty-}, \omega^{0} \in \mathbb{C}$.

Theorem 4.1. Let $P=(\lambda, y) \in \mathscr{K}_{n+1} \backslash\left\{P_{\infty+}, P_{\infty-}, P_{0}\right\},\left(x, t_{m}\right) \in M$, where $M \subseteq \mathbb{R}^{2}$ is open and connected. Suppose $u\left(x, t_{m}\right), v\left(x, t_{m}\right) \in C^{\infty}(M)$ satisfy the hierarchy of equations (2.7), and assume 
that $\lambda_{j}, 1 \leq j \leq 2 n+3$, in (3.12) satisfy $\lambda_{j} \in \mathbb{C} \backslash\{0\}$, and $\lambda_{j} \neq \lambda_{k}$ as $j \neq k$. Moreover, suppose that $\mathscr{D}_{\underline{\hat{\mu}}\left(x, t_{m}\right)}$ or equivalently, $\mathscr{D}_{\underline{\hat{v}}\left(x, t_{m}\right)}$, is nonspecial for $\left(x, t_{m}\right) \in M$. Then

$$
\frac{1}{u}=\frac{\omega^{\infty+}}{\omega^{0}} \frac{\theta\left(\underline{z}\left(P_{\infty+}, \underline{\hat{v}}\left(x, t_{m}\right)\right)\right) \theta\left(\underline{z}\left(P_{0}, \underline{\hat{\mu}}\left(x, t_{m}\right)\right)\right)}{\theta\left(\underline{z}\left(P_{\infty+}, \underline{\hat{\mu}}\left(x, t_{m}\right)\right)\right) \theta\left(\underline{z}\left(P_{0}, \underline{\hat{v}}\left(x, t_{m}\right)\right)\right)}+\frac{\omega^{0}}{\omega^{\infty-}} \frac{\theta\left(\underline{z}\left(P_{\infty-}, \underline{\hat{\mu}}\left(x, t_{m}\right)\right)\right) \theta\left(\underline{z}\left(P_{0}, \underline{\hat{v}}\left(x, t_{m}\right)\right)\right)}{\left.\theta\left(P_{\infty-}, \underline{\hat{v}}\left(x, t_{m}\right)\right)\right) \theta\left(\underline{z}\left(P_{0}, \underline{\hat{\mu}}\left(x, t_{m}\right)\right)\right)},
$$

$$
\frac{1-v}{1+v}=\frac{\left(\omega^{0}\right)^{2}}{\omega^{\infty+} \omega^{\infty-}} \frac{\theta\left(\underline{z}\left(P_{\infty+}, \underline{\hat{\mu}}\left(x, t_{m}\right)\right)\right) \theta\left(\underline{z}\left(P_{\infty-}, \underline{\hat{\mu}}\left(x, t_{m}\right)\right)\right) \theta^{2}\left(\underline{z}\left(P_{0}, \underline{\hat{v}}\left(x, t_{m}\right)\right)\right)}{\left.\theta\left(\underline{\hat{v}}\left(x, t_{m}\right)\right)\right) \theta\left(\underline{z}\left(P_{\infty-}, \underline{\hat{v}}\left(x, t_{m}\right)\right)\right) \theta^{2}\left(\underline{z}\left(P_{0}, \underline{\hat{\mu}}\left(x, t_{m}\right)\right)\right)} .
$$

Proof. According to Riemann's vanishing theorem [17,33], the definition and asymptotic properties of $\phi\left(P, x, t_{m}\right), \phi\left(P, x, t_{m}\right)$ has expression of the following type

$$
\phi\left(P, x, t_{m}\right)=N\left(x, t_{m}\right) \frac{\theta\left(\underline{z}\left(P, \underline{\hat{v}}\left(x, t_{m}\right)\right)\right)}{\theta\left(\underline{z}\left(P, \underline{\hat{\mu}}\left(x, t_{m}\right)\right)\right)} \exp \left(\int_{Q_{0}}^{P} \omega_{P_{0}, P_{\infty-}}^{(3)}(P)\right),
$$

where $N\left(x, t_{m}\right)$ is independent of $P \in \mathscr{K}_{n+1}, \hat{\mu}\left(x, t_{m}\right)=\left\{\hat{\mu}_{1}\left(x, t_{m}\right), \ldots, \hat{\mu}_{n+1}\left(x, t_{m}\right)\right\}, \underline{\hat{v}}\left(x, t_{m}\right)=$ $\left\{\hat{v}_{1}\left(x, t_{m}\right), \ldots, \hat{v}_{n+1}\left(x, t_{m}\right)\right\} \in \sigma^{n+1} \mathscr{K}_{n+1}$. Considering the asymptotic expansions of $\phi\left(P, x, t_{m}\right)$ near $P_{\infty \pm}$ and $P_{0}$, we have

$$
\begin{aligned}
\frac{1+v}{2 u} & =N\left(x, t_{m}\right) \omega^{\infty+} \frac{\theta\left(\underline{z}\left(P_{\infty+}, \underline{\hat{v}}\left(x, t_{m}\right)\right)\right)}{\theta\left(\underline{z}\left(P_{\infty+}, \underline{\hat{\mu}}\left(x, t_{m}\right)\right)\right)}, \\
\frac{2 u}{1-v} & =N\left(x, t_{m}\right) \omega^{\infty-} \frac{\theta\left(\underline{z}\left(P_{\infty-}, \underline{\hat{v}}\left(x, t_{m}\right)\right)\right)}{\theta\left(\underline{z}\left(P_{\infty}, \underline{\hat{\mu}}\left(x, t_{m}\right)\right)\right)}, \\
1 & =N\left(x, t_{m}\right) \omega^{0} \frac{\theta\left(\underline{z}\left(P_{0}, \underline{\hat{v}}\left(x, t_{m}\right)\right)\right)}{\theta\left(\underline{z}\left(P_{0}, \underline{\hat{\mu}}\left(x, t_{m}\right)\right)\right)},
\end{aligned}
$$

Then combining (4.28)-(4.30) yields (4.25) and (4.26).

\section{Conclusions}

In this paper, Finite genus solutions for Geng hierarchy are constructed, which are very important because they reveal inherent structure mechanism of solutions and describe the quasi-periodic behavior of nonlinear phenomenon or characteristic for the integrability of soliton equations. Moreover, they can be used to find multi-soliton solutions, elliptic function solutions, and others. However, we can't straighten the flows of the entire soliton hierarchy under the Abel-Jacobi coordinates, we will study it in the future.

\section{Acknowledgments}

This work was supported by the Key Scientific Research Projects of Henan Institution of Higher Education (No.17A110029) and Nanhu Scholars Program for Young Scholars of XYNU. 


\section{References}

[1] M. J. Ablowitz and H. Segur, Solitons and the inverse scattering transform (SIAM, Philadelphia, 1981).

[2] Y. Matsuno, Bilinear Transformation Method (Academic Press, New York, 1984).

[3] V. B. Matveev and M. A. Salle, Darboux transformations and solitons (Springer, Berlin, 1991).

[4] C. Rogers and W. K. Schief, Bäcklund and Darboux Transformations, Geometry and Modern Applications in Soliton Theory (Cambridge University Press, Cambridge, 2002).

[5] E. D. Belokolos, A. I. Bobenko, V. Z. Enolskii, A. R. Its and V. B. Matveev, Algebro-geometric approach to nonlinear integrable equations (Springer, Berlin, 1994).

[6] C. W. Cao, Y. T. Wu and X. G. Geng, Relation between the Kadomtsev-Petviashvili equation and the confocal involutive system, J. Math. Phys. 40 (1999) 3948-3970.

[7] M. L. Wang, Solitary wave solutions for variant Boussinesq equations, Phys. Lett. A, 199 (1995) 169172.

[8] E. Date and S. Tanaka, Periodic multi-soliton solutions of Korteweg-de Vries equation and Toda lattice, Progr. Theoret. Phys. Suppl. 59 (1976) 107-125.

[9] H. H. Dong, T. T. Chen, L. F. Chen and Y. Zhang, A new integrable symplectic map and the lie point symmetry associated with nonlinear lattice equations, J. Nonlinear Sci. Appl. 9 (2016) 5107-5118.

[10] X. G. Geng and Y. T. Wu, Finite-band solutions of the classical Boussinesq-Burgers equations, J. Math. Phys. 40 (1999) 2971-2982.

[11] W. X. Ma and J. H. Lee, A transformed rational function method and exact solutions to the $3+1$ dimensional Jimbo-Miwa equation, Chaos Solitons \& Fractals 42 (2009) 1356-1363.

[12] X. G. Geng and B. Xue, A three-component generalization of Camassa-Holm equation with N-peakon solutions, Adv. Math. 226 (2011) 827-839.

[13] G. Bluman and S. Kumei, On the remarkable nonlinear diffusion equation $(\partial / \partial x)[a(u+$ $\left.b)^{-2}(\partial u / \partial x)\right]-(\partial u / \partial t)=0$, J. Math. Phys. 21 (1980) 1019-1023.

[14] H. Wilhelmsson, Explosive instabilities of reaction-diffusion equations, Phys. Rev. A 36 (1987) 965966.

[15] O. P. Bhutani and K. Vijayakumar, On the isogroups of the generalised diffusion equation, Int. J. Eng. Sci. 28 (1990) 375-387.

[16] X. G. Geng, A new hierarchy of nonlinear evolution equations and corresponding finite-dimensional completely integrable systems, Phys. Lett. A 162 (1992) 375-380.

[17] F. Gesztesy and H. Holden, Soliton Equations and Their Algebro-Geometric Solutions. Vol. I: $(1+1)-$ Dimensional Continuous Models (Cambridge University Press, Cambridge, 2003).

[18] X. G. Geng and B. Xue, Soliton solutions and quasiperiodic solutions of modified Korteweg-de Vries type equations, J. Math. Phys. 51 (2010) 063516.

[19] X. G. Geng, L. H. Wu and G. L. He, Algebro-geometric constructions of the modified Boussinesq flows and quasi-periodic solutions, Physica D 240 (2011) 1262-1288.

[20] Y. Y. Zhai and X. G. Geng, Straightening out of the flows for the Hu hierarchy and its algebro-geometric solutions, J. Math. Anal. Appl. 397 (2013) 561-576.

[21] X. G. Geng, X. Zeng and B. Xue, Algebro-geometric solutions of the TD Hierarchy, Math. Phys. Anal. Geom. 16 (2013) 229-251.

[22] G. L. He, X. G. Geng and L. H. Wu, Algebro-geometric quasi-periodic solutions to the three-wave resonant interaction hierarchy, SIAM J. Math. Anal. 46 (2014) 1348-1384.

[23] F. Gesztesy, H. Holden, J. Michor and G. Teschl, Soliton Equations and Their Algebro-Geometric Solutions. Vol. II: (1+1)-Dimensional Discrete Models (Cambridge University Press, Cambridge, 2008).

[24] M. Antonowicz and A. P. Fordy, Coupled Harry Dym equations with multi-Hamiltonian structures, $J$. Phys. A 21 (1988) L269-L275.

[25] M. Antonowicz and A. P. Fordy, Factorisation of energy dependent Schrödinger operators: Miura maps and modified systems, Comm. Math. Phys. 124 (1989) 465-486.

[26] M.S. Alber, G.G. Luther and J.E. Marsden, Energy dependent Schrödinger operators and complex Hamiltonian systems on Riemann surfaces, Nonlinearity 10 (1997) 223-241.

[27] R. Camassa and D. D.Holm, An integrable shallow water equation with peaked solitons, Acta Math. Sinica 6 (1990) 35-41. 
[28] C. W. Cao, Stationary Harry-Dym's equation and its relation with geodesics on ellipsoid, Phys. Rev. A 36 (1987) 965-966.

[29] M. S. Alber and Y. N. Fedorov, Wave solutions of evolution equations and Hamiltonian flows on nonlinear subvarieties of generalized Jacobians, J. Phys. A 33 (2000) 8409-8425.

[30] M. S. Alber and Y. N. Fedorov, Algebraic geometrical solutions for certain evolution equations and Hamiltonian flows on nonlinear subvarieties of generalized Jacobians, Inverse Problems 17 (2001) 1017-1042.

[31] S. Abenda and Y. Fedorov, On the weak Kowalevski-Painlevé property for hyperelliptically separable systems, Acta Appl. Math. 60 (2000) 137-178.

[32] P. Vanhaecke, Integrable systems and symmetric products of curves, Math. Z. 227 (1998) 93-127.

[33] P. Griffiths and J. Harris, Principles of Algebraic Geometry (Wiley, New York, 1994).

[34] D. Mumford, Tata Lectures on Theta II (Birkhäuser, Boston, 1984).

[35] B. A. Dubrovin, Theta functions and nonlinear equations, Russian Math. Surveys (36) (1981) 11-92. 